\title{
Philosophiques
}

\section{Audrey Benoit, Trouble dans la matière, Paris, Éditions de la Sorbonne, coll. " Philosophies pratiques ", 2019, 359 pages}

\section{Capucine Mercier}

Volume 47, numéro 2, automne 2020

URI : https://id.erudit.org/iderudit/1075138ar

DOI : https://doi.org/10.7202/1075138ar

Aller au sommaire du numéro

Éditeur(s)

Société de philosophie du Québec

ISSN

0316-2923 (imprimé)

1492-1391 (numérique)

Découvrir la revue

Citer ce compte rendu

Mercier, C. (2020). Compte rendu de [Audrey Benoit, Trouble dans la matière, Paris, Éditions de la Sorbonne, coll. « Philosophies pratiques ", 2019,

359 pages]. Philosophiques, 47(2), 503-508. https://doi.org/10.7202/1075138ar d'utilisation que vous pouvez consulter en ligne.

https://apropos.erudit.org/fr/usagers/politique-dutilisation/ 


\section{Comptes rendus}

Audrey Benoit, Trouble dans la matière, Paris, Éditions de la
Sorbonne, coll. «Philosophies pratiques», 2019, 359 pages.

Les théories de Judith Butler sur la performativité du genre ont été assez mal reçues par le féminisme matérialiste français. Ce mauvais accueil, qui a de quoi surprendre, étant donné les nombreux points communs entre les travaux de Butler et ceux des féministes matérialistes, constitue le point de départ de l'ouvrage d'Audrey Benoit. L'autrice propose de réhabiliter la théorie butlérienne de la performativité du genre du point de vue matérialiste en l'inscrivant dans le cadre d'une épistémologie, principalement inspirée des travaux de Louis Althusser, qu'elle désigne sous le nom de "matérialisme discursif ». L'enjeu de Trouble dans la matière est en effet d'exposer comment la tradition matérialiste, à travers la figure centrale d'Althusser, mène précisément à l'approche épistémologique constructiviste mise en pratique par Butler. Ce faisant, Benoit esquisse un parallèle entre les démarches de Louis Althusser, de Michel Foucault et de Judith Butler, dans la mesure où chacun de ces auteurs considère le discours comme champ de construction des objets de la connaissance.

Cette exploration de la pensée de Butler sous l'angle de l'épistémologie vise à concilier féminisme queer et féminisme matérialiste, mais il offre aussi l'occasion d'aborder plus largement les nombreuses objections et réticences soulevées par la conception butlérienne du corps sexué comme production discursive. Il devient en effet clair que, pour Butler, toute connaissance possible de la matérialité du corps, comme de tout objet matériel, passe par le discours (soit, par des normes culturelles et sociales). Benoit oppose cette conception «matérialiste discursive» de la connaissance à une approche empiriste supposant un accès neutre et immédiat aux objets matériels, dont l'autrice révèle qu'elle constitue encore aujourd'hui le modèle épistémologique communément accepté. C'est donc parce qu'il met en cause ce cadre épistémologique que le traitement de la matérialité du corps chez Butler mystifie et choque de nombreux lecteurs et lectrices. Pourtant, comme le montre Benoit, si Butler aborde le corps sexué à travers le discours, elle n'en nie pas pour autant la matérialité; elle inverse plutôt, à la suite d'Althusser et de Foucault, l'ordre attendu entre le concept et l'objet matériel.

Benoit rappelle d'abord les grandes lignes de la théorie butlérienne du genre, et soulève certaines objections qui lui ont été faites. Elle se penche en particulier sur deux critiques matérialistes de Butler. La première, formulée par la philosophe américaine Nancy Fraser, reproche à Butler de confondre l'économique et le culturel dans son analyse. Fraser associe ainsi l'oppression genrée à des facteurs avant tout matériels, liés à la production et à la 
reproduction de la force de travail, considérant implicitement la culture comme un champ libre de tous rapports de pouvoir. Benoit souligne la convergence entre cette critique «multiculturaliste» et celle adressée par le féminisme matérialiste français au féminisme queer américain. Les féministes matérialistes ont en effet accueilli avec froideur les travaux de Butler sur le genre. Plutôt que de se reconnaître dans la démarche de la théoricienne américaine, qui vise comme la leur à mettre en cause la naturalité du genre et du sexe, des féministes d'envergure comme Nicole-Claude Mathieu et Christine Delphy s'en sont ouvertement dissociées. Elles considèrent que Butler, dans la lignée du poststructuralisme, se contente de s'intéresser aux «mots», mettant l'accent sur le discursif au détriment des injustices matérielles et économiques "réelles» qui perpétuent l'oppression. Les deux critiques de Butler ainsi évoquées par Benoît présentent donc de fortes ressemblances. Reprochant à Butler de ne s'intéresser qu'à la "culture" ou $\mathrm{au}$ "langage ", elles se fondent toutes deux sur la supposition d'une opposition fondamentale entre langage et réalité, culture et économie. Or, loin de délaisser la réalité matérielle du genre au profit du langage, la théorie de Butler a précisément pour vertu de dépasser une telle opposition. C’est ce que Benoit fait le pari de démontrer en répondant à ces objections, non du point de vue de la théorie butlérienne, mais du point de vue matérialiste luimême. En effet, le matérialisme n'implique pas nécessairement de privilégier la matière au discours et l'économie à la culture, comme le prouve l'épistémologie constructiviste développée par Althusser à partir d'une lecture originale de l'œuvre de Karl Marx. Le terme «matérialisme discursif » que Benoit emploie pour désigner cette approche épistémologique reflète d'ailleurs le dépassement de toute opposition naïve entre matière et discours.

Le cœur de l'ouvrage constitue une exposition de ce «matérialisme discursif». Benoit commence par exposer l'épistémologie constructiviste développée par Althusser à travers sa lecture du Capital. Pour Althusser, qui emprunte cette idée à Bachelard, la connaissance n'est pas assimilable à la «découverte» de faits; elle est plutôt la production de concepts. Althusser envisage par conséquent la théorie comme une forme de pratique; produisant des concepts, la théorie commande littéralement le mode de donation de l'objet. En effet, l'objet ne se donne pas au sujet de façon neutre, ni même à travers certaines interférences, comme le voudrait l'empirisme. Au contraire, il se présente toujours au sujet dans un cadre théorique (soit dans un cadre scientifique, soit dans celui, fondamentalement intéressé, de la simple doxa) qui détermine la façon dont il lui apparaît. La distinction stricte entre théorie et pratique n'a donc pas lieu d'être: la théorie a des effets pratiques sur ce qui apparaît comme objets de connaissance, et à l'inverse, la pratique contribue à la théorie puisqu'elle a une incidence directe sur les concepts produits. Voilà pourquoi le matérialisme marxien a pour Althusser un véritable caractère scientifique: en fournissant un nouveau cadre pratiquethéorique à la pensée, Karl Marx fait apparaître de nouveaux concepts et 
produit ainsi de nouvelles connaissances. Althusser illustre également ce principe de production de la connaissance à travers ce qu'il nomme la «lecture symptomale ", soit une lecture qui ne révèle pas le contenu caché d'un texte, mais produit plutôt de nouvelles connaissances à partir de celui-ci. C'est une telle lecture que Marx fait, selon Althusser, des économistes de son époque, et que lui-même fait de Marx.

Althusser maintient toutefois une certaine distinction entre l'objet réel et l'objet de la connaissance. Le processus de production du concept consisterait selon lui en l'abstraction de certaines caractéristiques d'un objet réel pour en faire un objet de connaissance, soit le seul auquel nous ayons accès. Un tel schéma, nous assure Benoit, n'a rien d'un dualisme kantien. Il permet plutôt à Althusser de définir la connaissance de façon fondamentalement non empirique, sans pour autant renoncer à parler de l'objet réel. Notons que des penseurs comme Foucault et Butler renonceront à cette notion d'objet réel, qui semble évoquer un schéma empiriste. Althusser lui-même reconnaît par ailleurs que l'objet réel non encore produit comme connaissance n'est "rien ", et que ce n'est qu'une fois approprié à travers une pratique et une théorie que l'objet a réellement une existence concrète: "Sans le langage et sans le droit, sans les rapports de production et les rapports idéologiques, aucune chose au monde n'est concrète pour l'homme» (p. 147).

En somme, les objets de connaissance se constituent dans l'environnement à la fois discursif et matériel, théorique et pratique qui est celui du sujet. Cet ensemble de rapports pratiques et théoriques qui déterminent la production d'objets de connaissance, Althusser le désigne sous le nom d'«idéologie». Il se différencie ainsi de Marx pour qui l'idéologie désigne plutôt une vision faussée de la réalité, une illusion que l'on pourrait dissiper. Abandonnant ce modèle de l'idéologie comme «représentation inversée » de la réalité, Althusser considère l'idéologie comme une condition de toute connaissance. En effet, on ne peut s'en abstraire pour avoir un contact immédiat avec le réel ou pour vérifier que la connaissance correspond bien à son objet. Toute idée d'adéquation entre la connaissance et le réel doit être abandonnée, car elle est issue d'une conception erronée, empiriste de la connaissance. Celle-ci ne peut être examinée que de façon immanente à l'idéologie, où le sujet est invariablement situé, et c'est donc par une réflexion interne et rigoureuse sur sa propre production qu'on peut juger de sa «scientificité»: "La scientificité revendiquée par le discours est appuyée sur un rapport réflexif du discours à sa propre production» (p. 104). Plutôt que de juger de la scientificité de la connaissance en fonction de son adéquation au réel, on doit donc lui poser «la question des conditions et du mécanisme de sa production» (p. 96). C'est le rôle que doit jouer pour Althusser la philosophie matérialiste: elle doit mettre en évidence les processus de production de la connaissance scientifique. En interrogeant la production de la catégorie de sexe, la théorie butlérienne semble donc remplir son office philosophique selon Althusser. 
Benoit cherche ensuite à étendre le concept de «matérialisme discursif » à la démarche de Foucault en montrant comment celle-ci met en cause la distinction entre le discursif et le matériel. L'autrice relève ainsi comment, dans L'Archéologie du savoir, la matérialité est présentée sous l'angle du discours, et le discours sous l'angle de la matérialité. L'histoire, par exemple, y est exposée comme "matérialité discursive composée par le travail de l'historien » (p. 207) à partir d'une masse documentaire à la fois discursive et matérielle. L'historien produit donc des concepts, plutôt qu'il ne découvre des faits bruts. Foucault brouille également la frontière entre le discursif et le matériel dans sa considération des conditions de possibilité d'énoncés (et donc de connaissances). Certaines de ces conditions en effet relèvent du langage (elles sont grammaticales, linguistiques) mais d'autres renvoient à des conditions matérielles concrètes diverses. En somme, le discours ne dépend pas uniquement, ni en premier lieu, du sujet, mais d'un ensemble de conditions théoriques et pratiques historiquement situées. Finalement, Benoit souligne que la démarche de Foucault dans cet ouvrage est une enquête sur la façon dont l'ordre "est ce qui rend possible toute signification» (p. 214). Influencé lui aussi par Bachelard, Foucault envisage ainsi la façon dont un certain cadre théorique (mais non indépendant de la pratique), l'epistémè, rend possibles certaines connaissances. À travers ces exemples, Benoit montre la proche parenté entre les pensées d'Althusser et de Foucault. Cette convergence s'explique entre autres par l'influence exercée sur chacun d'eux par les travaux (et, dans le cas de Foucault, l'enseignement) de Canguilhem. Celui-ci, en effet, pense le discours "comme milieu de production de ses objets plutôt que comme medium d'une signification idéelle» (p. 272). Pour Canguilhem, l'être humain est à concevoir plongé dans le discours comme l'être vivant dans son milieu, plutôt qu'employant le discours comme un outil. Héritier de cette vision, Foucault considère donc, comme Althusser, que «le discours n'a pas de dehors, au sens où il n'est que dehors. [Il] est le milieu général de la production des objets» (p. 229).

L'épistémologie du "matérialisme discursif» rend ainsi compte de l'imbrication nécessaire du concept et de la matière dans l'objet de connaissance. Si la matérialité est en elle-même l'une des conditions de possibilité de la connaissance, toute connaissance de la matière est en revanche conditionnée par des éléments relevant de la théorie et de la pratique, du discours et des conditions matérielles. Il est par conséquent illusoire de penser séparer le matériel du conceptuel: aucune matière brute ne s'offre à la connaissance, "on ne peut définir l'objet par son apparence immédiate et sensible: il faut nécessairement passer par le détour de son concept» (p. 110). En traitant un objet aussi concret que le corps sexué en termes discursifs, Butler n'attribue donc pas indûment aux mots le pouvoir de faire exister la matière. Elle travaille plutôt implicitement dans une épistémologie constructiviste semblable à celle que théorise Althusser: elle examine les conditions de la production discursive de la catégorie "sexe». Le discours a certes un ancrage matériel 
dans les corps; mais la matière ne peut être conçue comme une substance brute et objectivement observable, car elle est toujours saisie à travers des concepts et donc produite à travers le discours. Celui-ci n'est donc pas simplement plaqué sur la matière: il s'y ancre véritablement et la façonne en objet de connaissance.

Au terme de l'ouvrage, Benoit se tourne à nouveau vers la théorie féministe pour faire le rapprochement entre "la production par le discours de ses propres objets» (p. 302), théorisée par Althusser et Foucault, et la production de la matérialité sociale du sexe par le discours du genre dans la théorie butlérienne. C'est également l'occasion pour l'autrice de formuler sa propre critique de la théorie butlérienne. Benoit met en doute la pertinence de la notion de "performativité » que Butler emprunte à John Austin pour expliquer la production des catégories de genre et de sexe. En effet, la performativité austinienne centre l'analyse sur le locuteur individuel qui prononce l'énoncé performatif, plutôt que sur les conditions sociales qui conditionnent le pouvoir performatif de cet énoncé. Précisons que Butler élargit de beaucoup la notion de performativité, qui ne s'applique pour Austin qu'au langage; dans son œuvre, elle concerne toutes les pratiques productrices de signification, qu'elles soient ou non linguistiques. En conservant la notion de performativité, Butler hérite pourtant selon Benoit d'un souci du sujet individuel qui l'amène à chercher à expliquer la "capacité d'agir» [agency] des sujets, et en particulier leur capacité à résister au pouvoir. Ce faisant, elle «éloigne la problématique vers l'horizon de l'action individuelle, plutôt que de l'ancrer dans ses conditions sociales de possibilité, dont l'exploration butlérienne de l'assujettissement promettait l'étude» (p. 318). Benoit propose comme solution de «donner une réponse matérialiste au problème de la (re-)production sociale de la signification» en substituant à la notion de performativité butlérienne une réélaboration du concept d'idéologie au sens d'Althusser et de Foucault. Benoit revient finalement sur les objections concernant la matérialité du sexe évoquées en début d'ouvrage. Il apparaît maintenant que c'est la démarche épistémologique implicite dans l'œuvre de Butler qui choque ses critiques, car elle «bouscule le rapport attendu de la connaissance à son objet» (p. 324). Si le discours hétérosexiste produit le concept de "sexe", et à travers lui la matérialité du corps sexué, la solution est à la fois épistémologique et politique. Elle passe par la critique épistémologique des mécanismes de production de cette connaissance, et par des pratiques discursives qui produiront d'autres concepts et d'autres matérialités corporelles.

En somme, l'ouvrage de Benoit se distingue en amenant la théorie butlérienne sur le terrain de l'épistémologie, où la philosophe américaine ne s'est jamais explicitement engagée. L'autrice révèle ainsi de façon convaincante les présupposés épistémologiques constructivistes qui sous-tendent la critique des catégories de genre et de sexe, et explicite sous un angle neuf le lien entre matière et discours. La considération détaillée de la lecture de 
Marx chez Althusser, et des concepts d'histoire et de science chez Althusser et Foucault font toutefois de ce livre un traité d'épistémologie et de matérialisme davantage que de philosophie féministe. Plutôt que d'établir des liens tout au long du texte entre ces auteurs français et Butler, Benoit n'aborde directement la théorie féministe qu'au premier et dernier chapitre, de sorte qu'en somme ce livre plaira avant tout à celles et ceux qui s'intéressent à Althusser et à l'épistémologie marxiste et, de façon secondaire seulement, aux chercheuses en théorie féministe.

CAPUCINE MERCIER

University of New Mexico

Achille Mbembe, Brutalisme, Paris, La Découverte, 2020, 246 pages.

La Grand Theory sociologique et la philosophie de l'histoire ont été marquées dans les années 1980 et 1990 par l'emprunt du concept architectural de «postmodernité». En réaction au formalisme et au rationalisme modernistes, des architectes tels que Robert Venturi ou Michael Graves voulurent dans les années 1960 réhabiliter l'histoire et la culture vernaculaire dans l'ordre architectural. Il en résulta une certaine forme d'hybridation et d'éclectisme esthétiques, voire une «vitalité bordélique ${ }^{1}$ » (messy vitality), neutralisant les référentiels progressistes du modernisme. Des sociologues et des philosophes comme Fredric Jameson, Jean-François Lyotard ou Michel Freitag s'inspirèrent des théories architecturales postmodernistes afin de forger un concept du temps présent. Il fut l'objet de définitions diverses, voire contradictoires. On proposa même de voir dans la "postmodernité» une "hypermodernité ", c'est-à-dire non pas une sortie de la modernité, mais l'accentuation de ses tendances internes (Charles et Lipovetsky).

Le dernier essai d'Achille Mbembe, Brutalisme, réitère cet emprunt conceptuel à l'architecture. "J'emprunte le concept de brutalisme à la pensée architecturale ", écrit l'auteur dès la première ligne de son essai (p. 7). Mais, cette fois-ci, il ne s'agit plus de s'interroger sur la nature d'un processus transitoire, mais bien de décrire les résultats d'un accomplissement. Le brutalisme ayant été un mouvement architectural moderniste, cet emprunt suggère que la "brutalité » est moins le terme d'une éventuelle postmodernité que celui, pathologique, de la modernité elle-même; elle est en ce sens un hypermodernisme. La Cité radieuse de Le Corbusier est parfois citée comme un exemple d'architecture brutaliste: béton apparent, répétition d'éléments simples tels que des fenêtres et des pilotis, gigantisme et utopisme. Le brutalisme architectural agence des matériaux bruts, en particulier le béton, le

1. Robert Venturi, Complexity and Contradiction in Architecture, New York, The Museum of Modern Art, 1966, p. 16. 